klaren Ergebnissen. Er sucht die mannigfaltigen Verhältnisse, welche er beobachtet, durch die Annahme von bestimmten Hydraten zu erklären. Indem er die Voraussetzung macht, dass alle Elektrolyte gleiches äquivalentes Leitvermögen haben, muss er z. B. der Salzsäure die Formel $\mathrm{H}_{3} \mathrm{ClO}$ zuschreiben, und sie bei der Elektrolyse nach dem Schema $\mathrm{H}_{3}, \mathrm{ClO}$ zerfallen lassen. Warum die Chromsäure, welche kein bekanntes Hydrat bildet, gut leitet, und die Borsäure, deren Hydrat $\mathrm{H}_{3} \mathrm{BO}_{3}$ erst bei höherer Temperatur zersetzt wird, isolirt, vermag er nicht zu erklären.

Ich will mich nicht im Einzelnen auf eine Widerlegung dieser Anschauungen einlassen, denn ich hoffe, dass die einfachen und klaren Beziehungen, welche ich über die $\mathrm{Zu}-$ nahme des molecularen Leitvermögens mit der Verdünnung dargelegt habe, mich dessen überheben. Dass das Wasser für die Elektrolysirbarkeit der Säuren überhaupt von entscheidender Bedeutung ist, bedarf heute keines Beweises mehr. Dass aber durch die Annahme irgend welcher Hydrate die ganz allgemeine und ausnahmelose Gresetzmässigkeit, unter welcher der Einfluss des Wassers auf die Säuren, völlig unabhängig von der Natur der letzteren, steht, eine genügende Erklärung findet, wird der französische Forscher nach dem Durchlesen meiner Arbeit wohl selbst nicht behaupten wollen.

Riga, Polytechnikum, 1. Mai 1885.

\title{
Ueber die Einwirkung von Natriummerkaptid auf Phenylester;
}

von

\section{Richard Seifert.}

Die Alkohole haben, wie längst bekannt, viel schwächer saure Eigenschaften als die Merkaptane. Die Merkaptide bilden sich leichter und sind gegen Wasser beständiger als die Alkoholate. - In gleicher Weise stehen sich Phenole und Thiophenole gegenüber. 
Ueber die Beziehungen der Merkaptane zu den Phenolen habe ich hingegen in der Literatur keine Angaben gefunden. Beide Körperklassen bilden schon mit wässrigen Alkalien leicht salzartige Verbindungen. Um zu entscheiden, ob Phenol stärkere Säureeigenschaften als Merkaptan besitze, wurde eine concentrirte wässrige Lösung reinen Natriummerkaptids mit gepulvertem Phenol versetzt, welches sich leicht darin auflöst. Beim Verdünnen der so erhaltenen Lösung schied sich auf der Oberfläche sofort eine Schicht von Merkaptan ab. Dieses Resultat lässt aber noch nicht mit vollständiger Gewissheit auf den stärkeren Säurecharakter des Phenols schliessen, denn die Löslichkeit des Merkaptans in Wasser ist viel geringer als die des Phenols, und man könnte diese Reaction einfach für einen jener vielen Vorgänge ansehen, welche durch die „Schwerlöslichkeit" und andere "physikalische" Eigenschaften der Umsetzungsprodukte bedingt sind.

Dass jedoch in der That Phenol die stärkere Säure ist, also stärkere Affinität zu den Alkalien besitzt, habe ich durch eine Reaction bewiesen, welche den Hauptinhalt der vorliegenden Abhandlung ausmacht.

Ich habe gefunden, dass sich der Phenylester jeder beliebigen Säure mit Natriummerkaptid unter Abscheidung von Phenolnatrium in den Thioäthylester verwandelt:

$$
\begin{aligned}
\left(\mathrm{R}^{\mathrm{I}}-\mathrm{CO}\right)-\left(\mathrm{OC}_{6} \mathrm{H}_{5}\right) & +\mathrm{Na}-\left(\mathrm{S} \mathrm{C}_{2} \mathrm{H}_{5}\right)=\left(\mathrm{R}^{\mathrm{I}}-\mathrm{CO}\right)-\left(\mathrm{SO}_{2} \mathrm{H}_{5}\right) \\
& +\mathrm{Na}-\left(\mathrm{OC}_{6} \mathrm{H}_{5}\right) .
\end{aligned}
$$

Diese Reaction geht mit leicht schmelzbaren Estern schon beim einfachen Zusammenreiben mit dem Merkaptid ohne Zusatz eines Lösungsmittels vor sich. Hierbei steigert sich die Reactionswärme meist bis zum Schmelzen des Gemenges. Besser ist es, Aether als Verdünnungsmittel zu gebrauchen. $\mathrm{Da}$ sowohl Natriummerkaptid als auch Phenolnatrium in Aether kaum löslich sind, spielen Löslichkeitsverhältnisse bei dieser Umsetzung keine Rolle. Da die Reaction in ätherischer Verdünnung ohne grosse Wärmeentwicklung vor sich geht, so kann auch die Flüchtigkeit der Thioester, welche etwas grösser ist als die der Phenylester, nicht in Betracht kommen. Der Vorgang kann daher nur durch die Annahme erklärt 
werden, dass dem Complex $\left(\mathrm{C}_{6} \mathrm{H}_{5}-\mathrm{O}\right)$ - eine grössere Affinität zum Natrium und eine geringere zum Säurerest $\left(\mathrm{R}^{\mathrm{I}}-\mathrm{CO}\right)$-- innewohnt als dem Complex $\left(\mathrm{C}_{2} \mathrm{H}_{5}-\mathrm{S}\right)-$; oder genauer, dass die Affinität des Phenols zum Natrium vermehrt um die der Säuren zum Merkaptan grösser ist als die der Säuren zum Phenol plus der des Merkaptans zum Natrium. „Das heisst, Phenol ist saurer als Merkaptan, und letzteres basischer als Phenol."

I. Einwirkung von Natriummerkaptid auf Diphenylcarbonat.

Die eben besprochene Reaction wurde gelegentlich einer Reihe von Versuchen entdeckt, welche ich auf Veranlassung des Herrn Professor Dr. Schmitt zu dem Zwecke unternahm, die Synthesen der Salicylsäure näher zu erforschen. Bekanntlich hat W. Hents $\mathrm{ch} \mathrm{h}^{1}$ ) durch Erhitzen gleicher Moleküle Diphenylcarbonat und Natriumäthylat Salicylsäure erhalten gemäss der Gleichung:

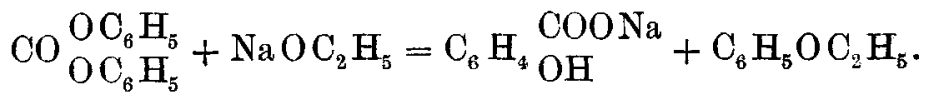

Als ich nun denselben Versuch mit Diphenylcarbonat und "Natriummerkaptid" wiederholen wollte, bemerkte ich, dass diese Körper nach kurzer Zeit schon in der Kälte, schneller bei gelinder Wärme auf einander einwirken.

Beim Zusammenreiben gleicher Moleküle von Diphenylcarbonat und Natriummerkaptid ${ }^{2}$ ) in einer ungefähr $50^{\circ}$ warmen Reibschale vurde das Gemisch nach kurzer Zeit unter bedeutender Wärmeentwickelung flüssig und beim darauffolgenden Erkalten langsam wieder fest. Der resultirende dicke Brei löste sich in Wasser unter Abscheidung eines Oeles, welches getrocknet und rectificirt wurde.

Das Thermometer stieg beständig von $190^{\circ}$ bis $310^{\circ}$. Von $300^{\circ}$ bis $310^{\circ}$ destillirte unverändertes Diphenylcarbonat. Der unter $300^{\circ}$ siedende Theil gab bei einer wiederholten

1) Dies. Journ. [2] 27, 44.

2) $\mathrm{Zu}$ diesen, wie allen übrigen Versuchen verwandte ich frisch bereitetes, im Wasserstoffstrom bei $80^{\circ}$ getrocknetes Merkaptid. 
Rectification abermals einen Rest von Diphenylcarbonat. Ich erhielt so im Ganzen ein Drittel der angewandten Menge des Diphenylcarbonats zurück.

In dem unter $300^{\circ}$ siedenden Antheil liessen sich schwefelhaltige Produkte nachweisen.

Die von dem Oele getrennte, alkalisch reagirende wässrige Lösung gab beim Ansäuren weder Merkaptan, noch Kohlensäure. noch Salicylsäure, dagegen eine beträchtliche Menge von Phenol, welches mit Aether ausgeschüttelt und durch einmalige Destillation vollkommen rein vom Siedepunkte $180^{\circ}$ bis $181^{\circ}$ und krystallisirt erhalten wurde.

Die gefundenen Thatsachen, dass nämlich über ein Drittel des verwandten Diphenylcarbonats nicht in Reaction gegangen, das Natriummerkaptid hingegen vollständig aufgebraucht war, dass sich ferner in dem Produkte Phenolnatrium und schwefelhaltige, hochsiedende Körper vorfanden, mussten zu dem Schlusse führen, dass sich Diphenylcarbonat und Natriummerkaptid im Sinne folgender Gleichung umsetzen:

$\mathrm{CO}_{\left(\mathrm{OC}_{6} \mathrm{H}_{5}\right)}^{\left(\mathrm{OC}_{6} \mathrm{H}_{5}\right)}+\underset{\mathrm{Na}\left(\mathrm{SC}_{2} \mathrm{H}_{5}\right)}{\mathrm{Na}\left(\mathrm{SC}_{2} \mathrm{H}_{5}\right)}=\mathrm{CO} \underset{\left(\mathrm{SC}_{2} \mathrm{H}_{5}\right)}{\left(\mathrm{SC}_{5} \mathrm{H}_{5}\right)}+2 \mathrm{Na}\left(\mathrm{OC}_{6} \mathrm{H}_{5}\right)$. Verlief die Reaction entsprechend dieser Gleichung, so musste die Hälfte des Diphenylcarbonats unangegriffen bleiben, da zur vollständigen Ueberführung des Diphenylcarbonats in Dithioäthylcarbonat „Zwei“ Moleküle Natriummerkaptid erforderlich sind.

In einer warmen Reibschale rieb ich nun „ein“ Molekül Diphenylcarbonat mit , „zwei“ Molekülen Natriummerkaptid innig zusammen. Der entstandene Brei wurde in Wasser gelöst, das abgeschiedene Oel von der wässrigen Lösung getrennt, der Rest des Oels mit Aether ausgeschüttelt und nach Abdestilliren des letzteren zur Hauptmenge hinzugefügt.

Die wässrige Lösung schied auf Zusatz von Säure grosse Mengen von Phenol ab, welches mit Aether extrahirt und durch einmalige Destillation krystallisirt erhalten wurde. Die Quantität entsprach nahezu der obigen Gleichung.

Das von der wässrigen Lösung gesonderte Oel wusch ich mit Wasser und trocknete es über Chlorcalcium. 
Bei der Destillation stieg nach einem sehr geringen Vorlauf das Thermometer schnell von $186^{\circ}-191^{\circ}$, dann langsam von $191^{\circ}-197^{\circ}$. Das Oel enthielt also kein Diphenylcarbonat. Das erhaltene Destillat erwies sich als fast reines Dithioäthylcarbonat, welches nach den Angaben von R. Schmitt ${ }^{1}$ ) in ganz reinem Zustande bei $196^{\circ}$ siedet.

Alle übrigen charakteristischen Eigenschaften dieses Körpers stimmten mit denen meines Produktes überein. Heisse alkoholische Kalilauge verseifte es schnell unter Bildung von Kaliumcarbonat und Kaliummerkaptid. Bei längerem Stehen mit alkoholischem Ammoniak zerlegte es sich in Harnstoff und Merkaptan. Der Harnstoff liess sich nach dem Eindampfen eines Theiles der Lösung an seinen charakteristischen Krystallen und seinem Nitrate erkennen. Ein anderer Theil der ammoniakalischen Lösung wurde mit Schwefelsäure angesäuert, destillirt und das Destillat in Quecksilberchloridlösung aufgefangen, in welcher sich der dicke, charakteristische Niederschlag von Chlorquecksilbermercaptid bildete.

Der Versuch hat also unzweifelhaft erwiesen, dass sich Diphenylcarbonat und Natriummerkaptid im Sinne der oben angeführten Gleichung umsetzen. Es entstand nun die Frage, ob diese Reaction nur dem Diphenylcarbonat eigenthümlich sei, oder ob sie den Phenylestern aller Säuren zukomme. Ich habe darauf bezügliche Versuche auf eine ganze Reihe von Phenylestern der verschiedensten Säuren ausgedehnt und gefunden, dass sich diese Umsetzung bei allen Phenylestern vollzieht.

Es kann aber vorkommen, dass leicht verseifbare Thioester, sobald sie nach Wasserzusatz der Einwirkung einer wässrigen alkalischen Lösung ausgesetzt sind, secundär zersetzt werden. Dieser Fall scheint bei dem Thioäthylformiat einzutreten.

Im Folgenden sei mir erlaubt, die einzelnen Ergebnisse mitzutheilen.

1) Ber. Berl. chem. Ges. 1, 166. 


\section{Phenylformiat und Natriummerkaptid.}

Phenylformiat lässt sich nicht aus Formamid und Phenol darstellen, etwa wie nach Guareschi ${ }^{1}$ ) Phenylacetat aus Acetamid und Phenol. Selbst nach mehrtägigem Kochen hatte sich keine Spur eines in verdünnten Alkalien unlöslichen Produktes gebildet. Nencki erwähnt in seiner Abhandlung ${ }^{2}$ ), ,über die Verbindungen der Fettsäuren mit Phenolen", dass man aus einer Säure durch Erwärmen mit Phenol und Phosphoroxychlorid ihren Phenylester erhalten könne. Kurz darauf bereitete Rasińsky ${ }^{3}$ ) auf diesem Wege einige Phenylester.

Auch ich habe mich zur Herstellung fast aller in dem noch folgenden Theile der Arbeit benutzten Phenylester dieser schönen Methode bedient und dabei gefunden, dass man nahezu quantitative Ausbeuten und sehr reine Producte erhält, wenn man mit einem nicht zu starken Ueberschuss von der theoretisch nöthigen Menge Phosphoroxychlorid arbeitet und die Reaction bei möglichst gemässigter Temperatur sehr langsam ausführt.

Ein Gemisch gleicher Moleküle Ameisensäure und Phenol wurde auf $80^{\circ}$ erwärmt und allmälig mit einem Drittel-Molekül Phosphoroxychlorid behandelt, das flüssige Produkt nach Beendigung der Salzsäureentwickelung in kalte, verdünnte Sodalösung gegossen, das ausgeschiedene, schwach röthlich gefärbte Oel mit verdünnter Natronlauge und darauf mit Wasser gewaschen.

Ich erhielt so Phenylformiat als ölige Flüssigkeit von schwachem Geruche, welcher an den des Diphenylcarbonats erinnerte. Es löst sich erst nach einiger Zeit in kalter verdünnter, schneller in concentrirter Natronlauge. Nach dem Trocknen im Vacuum über Schwefelsäure siedete das Oel bei $179^{\circ}-180^{\circ}$, aber unter starker Zersetzung, denn das Destillat war nur noch zum Theil in Alkalien unlöslich.

1) Ann. Chem. Pharm. 171, 142.

2) Dies. Journ. [2] 25, 282.

3) Das. [2] 26, 62 . 
Das weder rein noch trocken erhaltene Phenylformiat behandelte ich in ätherischer Lösung mit Natriummerkaptid. Wasser löste aus dem Produkte Phenolnatrium und schied eine ätherische Schicht ab. Aus dieser erhielt ich nur sehr geringe Mengen einer zwischen $80^{\circ}$ und $90^{\circ}$ siedenden Flüssigkeit, welche das Merkaptan an widrigem Geruche übertraf. Es scheint hiernach, dass Thioäthylformiat schon in der Kälte von wässrigen Alkalien verseift wird.

ШI. Thioäthylacetat aus Phenylacetat.

Nach mehreren Versuchen hielt ich folgendes Verfahren ein. Reines Phenylacetat (ein Molekül) wurde in einer gelinde erwärmten Reibschale allmälig mit etwas mehr als einem Molekül Natriummerkaptid zusammengerieben, wobei sich die Masse stark erwärmte. Das Produkt löste ich in eiskaltem Wasser, trennte das abgeschiedene Oel und trocknete es über Chlorcalcium. Nach mehrmaligem Rectificiren siedete fast die ganze Menge zwischen $116^{\circ}-118^{\circ}$. Die vom Oele gesonderte wässrige Lösung schied auf Säurezusatz Phenol ab. Spätere Versuche mit anderen Estern machen es wahrscheinlich, dass es auch hier besser ist, das Zusammenreiben unter Aetherzusatz vorzunehmen und das Gemisch vor der Behandlung mit Wasser kurze Zeit sich selbst zu überlassen.

Die erhaltene Flüssigkeit vom Siedepunkt $116^{\circ}-118^{\circ}$ zeigte alle Eigenschaften des Thioäthylacetats, welches bei $116^{\circ}$ sieden soll.

Bei der Verbrennung mit Bleichromat und Kupferoxyd gaben 0,2019 Grm. Substanz 0,33505 Grm. Kohlensäure und 0,14635 Grm. Wasser. Bei einer Schwefelbestimmung nach Carius gaben 0,1071 Grm. Substanz 0,2391 Grm. Bariumsulfat.

Für $\mathrm{CH}_{3}-\mathrm{COSO}_{2} \mathrm{H}_{5}$ berechnet.

$\begin{array}{ccc}\mathrm{C} & 46,2 & \mathbf{4 5 , 9} \\ \mathrm{H} & 7,7 & 8,05 \\ \mathrm{~S} & 30,77 & 30,66\end{array}$

Die Reaction verläuft also gemäss der Gleichung:

$$
\begin{aligned}
\mathrm{OH}_{3} \cdot \mathrm{CO}\left(\mathrm{OC}_{6} \mathrm{H}_{5}\right) & +\mathrm{Na}\left(\mathrm{SC}_{2} \mathrm{H}_{5}\right)=\mathrm{CH}_{3} \mathrm{CO}\left(\mathrm{SC}_{2} \mathrm{H}_{5}\right) \\
& +\mathrm{Na}\left(\mathrm{OC}_{6} \mathrm{H}_{5}\right) .
\end{aligned}
$$


Es kann nicht zweifelhaft sein, dass sich die Phenylester anderer Fettsäuren analog verhalten.

Die folgenden Versuche beziehen sich auf die Phenylester aromatischer Säuren und mehrbasischer Fettsäuren, wie Bernsteinsäure, Citronensäure, Benzoësäure und Salicylsäure.

\section{Dithioäthylsuccinat aus Diphenylsuccinat.}

Diphenylsuccinat und Natriummerkaptid setzen sich sehr glatt um in Dithioäthylsuccinat und Phenolnatrium:

$$
\begin{aligned}
\mathrm{C}_{2} \mathrm{H}_{4}\left(\mathrm{COOC}_{6} \mathrm{H}_{5}\right)_{2} & +2 \mathrm{NaSC}_{2} \mathrm{H}_{5}=\mathrm{C}_{2} \mathrm{H}_{4}\left(\mathrm{COSC}_{2} \mathrm{H}_{5}\right)_{2} \\
& +2 \mathrm{NaOC}_{6} \mathrm{H}_{5} .
\end{aligned}
$$

Ein Molekiul Diphenylsuccinat ( $42 \mathrm{Grm}$.) wurde mit absolutem Aether und etwas mehr als zwei Molekülen Natrium. merkaptid (30 Grm.) bei gewöhnlicher Temperatur zusammengerieben. Die ganze Masse löste sich zur dünnen Flüssigkeit, welche nach kurzer Zeit Phenolnatrium abschied. Wärmeentwickelung war nicht bemerkbar. Der resultirende dicke Brei gab auf Wasserzusatz eine ätherische Lösung von Dithioäthylsuccinat und eine wässrige von Phenolnatrium. Aus der letzteren konnten nach dem Ansäuern 25 Grm. Phenol extrahirt werden. Nach der obigen Gleichung sollen $29 \mathrm{Grm}$. erbalten werden. Die von der wässrigen Lösung getrennte Aetherschicht wurde mehreremale mit Wasser geschüttelt, bis dieses nicht mehr alkalisch reagirte, dann getrocknet, und der Aether abdestillirt. Das zurückbleibende Oel siedete nach zweimaligem Trocknen und Rectificiren mit Ausnahme eines sehr geringen Vorlaufes vollständig von $269^{\circ}$ bis $271^{\circ}$ und bestand aus Dithioäthylsuccinat.

Das Dithioäthylsuccinat, welches bis jetzt noch nicht dargestellt wurde, bildet in reinem Zustande ein wasserhelles Oel von lauchartigem Geruche. Es färbt sich nach einiger Zeit gelblich und siedet unter ganz geringer Zersetzung von $270^{\circ}$ bis $271^{\circ}$.

Bei einer Schwefelbestimmung nach Carius gaben 0,1329 Grm. Substanz 0,2971 Grm. Bariumsulfat.

Der Körper $\mathrm{C}_{2} \mathrm{H}_{4}\left(\mathrm{COSC}_{2} \mathrm{H}_{5}\right)_{2}$ enthält: Gefunden.

$$
\mathrm{S} \quad 31,0 \%
$$




\section{Trithioäthylcitrat aus Triphenylcitrat.}

Da die Citronensäure nicht leicht vollständig wasserfrei zu erhalten ist, so benutzte ich zur Bereitung des Triphenylcitrats fein zerriebenes, bei $170^{\circ}$ getrocknetes, neutrales Natriumcitrat, mischte dasselbe in einer Kochflasche mit drei Molekülen Phenol, erwärmte den mit Bunsenventil versehenen Kolben auf einem Wasserbade und gab nach und nach etwas über ein Molekül Phosphoroxychlorid hinzu. Bei Anwendung von $60 \mathrm{Grm}$. Natriumcitrat hörte die Salzsäureentwickelung nach 15-18 Stunden auf. Das Produkt war ein dicker, braungelber Syrup, welcher auf Zusatz von Wasser ein Oel abschied. Dieses Oel erstarrte beim Zerreiben mit erneutem Wasser zu einer hellgelben Krystallmasse. Diese wurde mit Sodalösung behandelt, mit Wasser gewaschen, getrocknet und aus absolutem Alkohol mehrmals umkrystallisirt.

Das Triphenylcitrat $\mathrm{C}_{3} \mathrm{H}_{5} \mathrm{O}\left(\mathrm{COOC}_{6} \mathrm{H}_{5}\right)_{3}$ bildet farblose Nadeln vom Schmelzpunkte $124,5^{\circ}$, ist unlöslich in Wasser, sehr leicht löslich in absolutem heissen Alkohol, etwas weniger in kaltem.

Bei der Elementaranalyse gaben 0,1209 Grm. Substanz 0,05395 Grm. Wasser und 0,3031 Grm. Kohlensäure.

Der Formel $\left(\mathrm{C}_{3} \mathrm{H}_{5} \mathrm{O}\left(\mathrm{COOC}_{6} \mathrm{H}_{5}\right)_{3}\right.$

\begin{tabular}{ccc}
\multicolumn{2}{c}{ entsprechen: } & Gefunden. \\
$\mathrm{C}$ & $68,57 \%$ & $68,374 \%$ \\
$\mathrm{H}$ & $4,76 \%$ & $4,96 \%$
\end{tabular}

Triphenylcitrat (ein Molekül) wurde mit absolutem Aether und Natriummerkaptid (etwas über drei Moleküle) innig zusammengerieben. Der dünne Brei ging beim Aufbewahren in einer gut verkorkten Kochflasche in eine klare Lösung über, aus welcher das darin enthaltene Phenolnatrium selbst nach zweitägigem Stehen nicht ausfiel. Wasser löste aber aus der Masse das Phenolnatrium unter Abscheidung einer ätherischen Schicht, welche bis zur neutralen Reaktion gewaschen und über Chlorcalcium getrocknet wurde. Nach dem Abdestilliren des Aethers versuchte ich, das zurückbleibende Oel im Vacuum zu fractioniren. Dabei zersetzte 
es sich jedoch unter starker Verkohlung vollständig. Die Unmöglichkeit, diesen Thioester zu destilliren, erschwerte seine Reindarstellung ungemein. Jedoch war das nach dem Abdestilliren des Aethers im Vacuum aus einem Oelbade von $110^{\circ}$ zurückbleibende Oel, nachdem ich es noch über Chlorcalcium getrocknet hatte, fast reines Thioäthylcitrat.

Trithioäthylcitrat ist ein schwach gelbrothes Oel, welches merkaptanartig riecht und mit Triäthylcitrat den intensiv bittern Geschmack gemein hat.

Bei einer Schwefelbestimmung nach Carius lieferten 0,13125 Grm. Subtanz 0,2705 Grm. Bariumsulfat.

Die Verbindung $\mathrm{C}_{3} \mathrm{H}_{5} \mathrm{O}\left(\operatorname{COSC}_{2} \mathrm{H}_{5}\right)_{8}$ enthält:

Gefunden.

S $29,6 \% \quad 28,27 \%$

Trotz dieser nicht ganz stimmenden Analyse, zu welcher ja eine kaum einer Reinigungsoperation unterworfene Substanz benutzt worden war, kann kein Zweifel herrschen, dass auch in diesem Falle die Reaction nach der Gleichung verlief

$$
\begin{aligned}
\mathrm{C}_{3} \mathrm{H}_{5} \mathrm{O}\left(\mathrm{COOC}_{6} \mathrm{H}_{5}\right)_{3} & +3 \mathrm{NaSC}_{2} \mathrm{H}_{5}=\mathrm{C}_{3} \mathrm{H}_{5} \mathrm{O}\left(\mathrm{COSC}_{2} \mathrm{H}_{5}\right)_{3} \\
& +3 \mathrm{NaOC}_{6} \mathrm{H}_{5} .
\end{aligned}
$$

VI. Thioäthylbenzoat aus Phenylbenzoat.

Ein Molekül des reinen Phenylbenzoats, nach dem Verfahren von Nencki und Rasińsky dargestellt, wurde mit etwas mehr als einem Molekül Natriummerkaptid und absolutem Aether zusammengerieben. Die Reaktion verlief langsamer, als in den vorhergehenden Fällen und ohne bemerkbare Wärmeentwicklung. Binnen wenigen Minuten war die ganze Masse in eine Lösung übergegangen, aus welcher sich bald festes Phenolnatrium abschied. Der Brei wurde noch einige Zeit sich selbst tiberlassen, dann mit Wasser behandelt, das abgeschiedene Oel getrennt, gewaschen und über Chlorcalcium getrocknet. In der wässrigen Lösung befand sich Phenolnatrium und das überschüssige Natriummerkaptid. Das trockne Oel destillirte nach einem geringen Vorlaufe von Aether bei $241^{\circ}-243^{\circ}$. Es zeigte den charakteristischen 
Geruch und alle sonstigen Eigenschaften des von Engel. hardt, Latschinow und Malyschew ${ }^{\mathrm{I}}$ ) schon beschriebenen, auf andere Weise erbaltenen "Thioäthylbenzoats".

Hieraus geht hervor, dass auch die Phenylester aromatischer Säuren die Gruppe $\left(\mathrm{C}_{6} \mathrm{H}_{5}-\mathrm{O}\right)-$ gegen $\left(\mathrm{C}_{2} \mathrm{H}_{5}-\mathrm{S}\right)-$ austauschen:

$$
\begin{aligned}
\mathrm{C}_{6} \mathrm{H}_{5} \cdot \mathrm{COOC}_{6} \mathrm{H}_{5}+\mathrm{NaSC}_{2} \mathrm{H}_{5} \\
=\mathrm{C}_{6} \mathrm{H}_{5} \cdot \operatorname{CosC}_{2} \mathrm{H}_{5}+\mathrm{NaOC}_{6} \mathrm{H}_{5} .
\end{aligned}
$$

VII. Einwirkung ron Natriummerkaptid auf Phenylsalicylat und Phenylmethylsalicylat. Thioäthylmethylsalicylat.

Zur Darstellung von Phenylsalicylat schmolz ich 69 Grm. Salicylsäure (1 Molekül) mit 48 Grm. Phenol (1 Molekül) bei ungefähr $135^{\circ}$ zusammen und setzte in kleinen Portionen $28 \mathrm{Grm}$. Phosphoroxychlorid (etwas über $1 / 3$ Molekül) hinzu. Sobald die Salzsäureentwicklung im Gange war, liess ich das Gemisch auf $125^{\circ}$ bis $120^{\circ}$ erkalten und erhitzte es während der ganzen folgenden Reaktion nicht höher. Gegen Ende des Processes setzte sich am Boden des Gefässes eine zähe Schicht von Phosphorsäure fest, von welcher der Phenylester in viel kaltes Wasser abgegossen wurde. Er schied sich zunächst als schwach röthlich gefärbtes Oel ab, welches durch Behandeln mit erneutem Wasser zu einer festen Krystallmasse erstarrte. Diese rieb ich mit verdünnter Sodalösung zusammen, bis sich keine Kohlensäure mehr entwickelte, also alle noch beigemengte Salicylsäure gelöst war. Hierauf wurden die Krystalle abfiltrirt, mit Wasser gewaschen und aus starkem Weingeist umkrystallisirt. Ich erhielt statt $107 \mathrm{Grm}$. $99 \mathrm{Grm}$. fast weisses Phenylsalicylat. Beim Erkalten der alkoholischen Lösung scheidet sich der Ester zuerst in Form kleiner Oeltröpfchen aus, welche bei der Berührung mit einem Krystall des Körpers und beim Abkühlen krystallinisch erstarren.

Eine Elementaranalyse gab aus 0,1554 Grm. Subst. 0,4128 Grm. Kohlensäure und 0,0681 Grm. Wasser.

1) Zeitsehr. Chem. 1868, S. 353. 


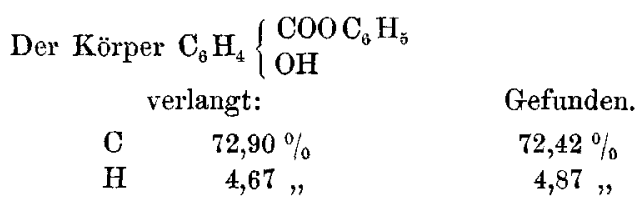

Das Phenylsalicylat ist noch nicht dargestellt worden. Es bildet farblose, rhombische Tafeln (aus Methylalkohol) vom Schmelzpunkt $42^{\circ}$ bis $42,5^{\circ}$, ist unlöslich in Wasser, sehr leicht löslich in heissem, weniger in kaltem Methylund Aethylalkohol. Der Ester ist ferner leicht löslich in Aether. In festem Zustande ist er fast geruchlos, dagegen besitzt die verdünnte alkoholische Lösung einen an Wintergrünöl erinnernden Geruch. Das Phenylsalicylat zeigt sehr schön die Erscheinung der Ueberschmelzung. Lässt man geschmolzenen Ester langsam erkalten, so bleibt er flüssig und wird erst beim Reiben mit einem Glasstabe oder durch hineingeworfene Krystalle unter bedeutender Temperaturerhöhung wieder fest. Seine alkoholische Lösung wird durch Eisenchlorid violett gefärbt.

Kochende Natronlange verseift den Ester sofort, während beim Zusammenreiben mit kalter, concentrirter Natronlauge ein dicker Brei von Natriumsalicylsäurephenylester, $\mathrm{C}_{6} \mathrm{H}_{4} \mathrm{CONa}_{6} \mathrm{H}_{5}$ entsteht. Dieses Natriumsalz löst sich in Wasser und Alkohol. Die kalte wässrige Lösung scheidet auf Säurezusatz unverändertes Phenylsalicylat $a b$, zersetzt sich aber beim Kochen sehr leicht in Phenol und Natriumsalicylat:

$$
\mathrm{C}_{6} \mathrm{H}_{4} \underset{\mathrm{ONa}}{\mathrm{COOC}_{6} \mathrm{H}_{5}}+\mathrm{H}_{2} \mathrm{O}=\mathrm{C}_{6} \mathrm{H}_{4} \underset{\mathrm{OH}}{\mathrm{COONa}}+\mathrm{C}_{6} \mathrm{H}_{5} \mathrm{OH} \text {. }
$$

Um das Phenylsalicylat in Thioäthylsalicylat überzuführen, rieb ich es mit absolutem. Aether und der äquivalenten Menge Natriummerkaptid zusammen. Die Mischung gestand zu einem dicken Brei, welcher nicht wie in den früheren Fällen in eine Lösung überging, sondern nichts weiter als Natriumphenylsalicylat, $\mathrm{C}_{6} \mathrm{H}_{4} \mathrm{COOC}_{6} \mathrm{H}_{5}$ und Merkaptan enthielt. 
Die dem Merkaptan überlegene Säurenatur des Phenols hatte sich auch hier geltend gemacht, aber nicht durch Bildung von Phenolnatrium und Thioäthylsalicylat, sondern in der Weise, dass das substituirte Phenol $\left(\mathrm{C}_{6} \mathrm{H}_{5} \mathrm{OCO} . \mathrm{O}_{6} \mathrm{H}_{4}\right) \mathrm{OH}$ seinen Hydroxylwasserstoff gegen das Metall des Merkaptids austauschte:

$$
\begin{aligned}
\mathrm{C}_{6} \mathrm{H}_{4} \underset{\mathrm{OH}}{\mathrm{COOC}_{6} \mathrm{H}_{5}}= & \mathrm{NaSC}_{2} \mathrm{H}_{5}=\mathrm{C}_{6} \mathrm{H}_{4} \underset{\mathrm{ONa}}{\mathrm{COOC}_{6} \mathrm{H}_{5}} \\
& +\mathrm{C}_{2} \mathrm{H}_{5} \mathrm{SH} .
\end{aligned}
$$

Da das entstandene Natriumsalz in Aether vollständig unlöslich ist, hatte der Zusatz eines zweiten Moleküls Natriummerkaptid keine Wirkung. Auch der Ersatz des Aethers durch Alkohol führte nicht zu dem gewünschten Thioäthylsalicylat. Es blieb daher nur übrig, die Reaktion an einem Phenylsalicylat zu studiren, dessen Hydroxylwasserstoff durch ein Alkyl ersetzt war.

Ich wählte Phenylmethylsalicylat und stellte dieses aus Methylsalicylsäure und Phenol ganz analog dem Phenylsalicylat dar. Die Reaktion begann hier bei $125^{\circ}$ und wurde grösstentheils bei $110^{\circ}-115^{0}$ weitergeführt. Das Produkt, in der beim Phenylsalicylat beschriebenen Weise behandelt, erstarrte nicht so schnell wie dort zu einem Krystallbrei. Der getrocknete rohe Ester wurde aus wenig absolutem Alkohol umkrystallisirt, und der in der Mutterlauge bleibende Rest mit Wasser gefällt.

Phenylmethylsalicylat, $\mathrm{C}_{6} \mathrm{H}_{4}^{\mathrm{COCH}_{3}}{ }^{\mathrm{OOOC}_{5}}$, bildet weisse sechsseitige, wahrscheinlich monokline Prismen (aus absolutem Alkohol) vom Schmelzpunkte 59 ${ }^{\circ}$, ist unlöslich in Wasser, sehr leicht löslich in warmem, schwerer in kaltem Methyl- und Aethylalkohol, sehr leicht löslich in Aether.

Bei der Elementaranalyse gaben 0,1722 Grm. Subst. 0,0851 Grm. Wasser und 0,46755 Grm. Kohlensâure.

Die Verbindung $\mathrm{C}_{8} \mathrm{H}_{4}\left\{\begin{array}{l}\mathrm{COOO}_{6} \mathrm{H}_{5} \\ \mathrm{OCH}_{3}\end{array}\right.$

$$
\begin{array}{lr}
\multicolumn{2}{c}{\text { verlangt: }} \\
\mathrm{C} & 73,7 \% \\
\mathrm{H} & 5,3 \%
\end{array}
$$

Gefunden.$$
74,0 \%
$$$$
5,5,
$$ 


$$
\text { Thioäthylmethylsalicylat. }
$$

Reines Phenylmethylsalicylat wurde mit absolutem Aether und etwas mehr als der äquivalenten Menge Natriummerkaptid zusammengerieben. Beim Aufbewahren des Breies in einem gut verschlossnen Kölbchen trat nach einiger Zeit vollständige Lösung und darauf eine geringe Abscheidung von Phenolnatrium ein. Die Lösung wurde in Wasser von $0^{0}$ gegossen, da die Ester der Salicylsäure durch Alkalien bekanntlich schon bei geringem Erwärmen verseift werden, das ausgeschiedene Oel mit Wasser bis zur neutralen Reaktion gewaschen und über Chlorcalcium getrocknet. Durch Ausschütteln der wässrigen Lösung mit Aether gewann ich noch etwas Oel und fügte es zur Hauptmenge.

Die wässrige Lösung enthielt Phenolnatrium und das überschüssig angewandte Natriummerkaptid. Das trockne Oel wurde bei $80 \mathrm{Mm}$. Druck rectificirt. Schon nach wenig überdestillirten Tropfen stieg das Thermometer auf $196^{\circ}$, dann langsam bis $199^{\circ}$.

Thioäthylmethylsalicylat ist ein wasserhelles Oel, welches analog dem Thioäthylbenzoat nach Merkaptan und zugleich aromatisch riecht. Es siedet bei $80 \mathrm{Mm}$. Druck von $197^{\circ}$ bis $198^{\circ}$ unter minimaler Zersetzung.

Bei einer Schwefelbestimmung mit Soda und Kaliumchlorat nach Kolbe gaben 0,1247 Grm. Substanz 0,1497 Grm. Bariumsulfat.

Die Verbindung $\mathrm{C}_{6} \mathrm{H}_{4}\left\{\begin{array}{l}\mathrm{COSC}_{9} \mathrm{H}_{5} \\ \mathrm{OCH}_{3}\end{array}\right.$

$$
\begin{array}{cc}
\text { s verlangt: } & \text { Gefunden. } \\
\text { S } 16,33 \% & 16,48 \%
\end{array}
$$

Die Reaction war also auch hier nach der Gleichung:

$$
\begin{aligned}
& \mathrm{CH}_{3} \mathrm{O} \cdot \mathrm{C}_{6} \mathrm{H}_{4} \cdot \mathrm{COOC}_{6} \mathrm{H}_{5}+\mathrm{NaSC}_{2} \mathrm{H}_{5} \\
= & \mathrm{CH}_{3} \mathrm{O} \cdot \mathrm{C}_{6} \mathrm{H}_{4} \cdot \mathrm{COSO}_{2} \mathrm{H}_{5}+\mathrm{NaOC}_{6} \mathrm{H}_{5}
\end{aligned}
$$

verlaufen.

Wegen der leichten Zugänglichkeit der Phenylester bietet die in vorliegender Abhandlung beschriebene Reaction ${ }^{\mathbf{1}}$ )

5) Dieselbe hat einige Aehnlichkeit mit der von Kekulé (Zeitschr. Chem. 1867, S. 196) und später von Weselsky (Ber. Berl. chem. 
eine sehr bequeme Methode zur Herstellung von Thioestern besonders solcher Säuren, deren Chloride schwer darzustellen sind.

Davon abgesehen bildet das Hauptergebniss dieser Arbeit die Erkenntniss, dass Phenol saurer ist als Merkaptan.

"Alkohol, Merkaptan, Phenol, Thiophenol" bilden somit eine Reihe, in welcher von links nach rechts die basischen Eigenschaften ab-, die sauren zunehmen."

Diese Reihe führt zu einigen Folgerungen.

Zum Beispiel werden bei gewöhnlicher Temperatur "Phenolate" $\left[\mathrm{C}_{6} \mathrm{H}_{5} \mathrm{O}\right]-\stackrel{\mathrm{I}}{\mathrm{M}}$ auf die "Ester der Alkohole" $[\mathrm{R}-\mathrm{CO}]-\left[\mathrm{OC}_{2} \mathbf{H}_{5}\right]$ (oder allgemeiner $[\mathrm{R}-\mathrm{CO}]-[-\mathrm{O} \mathrm{Alk}$.$] )$ nicht einwirken; denn das saure Phenol ist schon mit der Base, der basischere Alkohol schon mit der Säure verbunden. Die in diesem Falle einzig mögliche Umsetzung, nämlich diejenige, dass die Säure des Esters das Alkali an sich reisst, nnd gleichzeitig Phenyläther gebildet werden, findet nur mit den Estern der Mineralsäuren und auch da nur bei höherer Temperatur statt: Darstellung von Phenyläthern aus Phenolnatrium und Alkylhalogenen oder Alkylkaliumsulfat.

Dagegen werden schon bei gewöhnlicher Temperatur "Phenolate" "die Ester des Thiophenols" in "Thiophenolat" und „Ester des Phenols" umsetzen. Ferner werden Merkaptide ausser den Estern des Phenols auch die des Thiophenols zerlegen, und Alkoholate die Ester des Merkaptans, Phenols und Thiophenols.

Dass diese letztere Folgerung kein Fehlschluss ist, prüfte ich noch an dem Verhalten des Natriumäthylats zum Diphenylcarbonat.

VIII. Diphenylcarbonat und Natriumäthylat.

Ich erhielt aus diesen Körpern in der That Diäthylcarbonat und Phenolnatrium gemäss der Gleichung:

Ges. 2, 250) untersuchten „Verseifung" der Phenylester durch HKS, wobei neben Phenolkalium die Kaliumsalze der Thiosäuren entstehen, z. B.:

$$
\mathrm{CH}_{3} \mathrm{COOC}_{6} \mathrm{H}_{5}+2 \mathrm{~K} \cdot \mathrm{SH}=\mathrm{CH}_{3} \mathrm{CO}-\mathrm{SK}+\mathrm{C}_{6} \mathrm{H}_{5} \mathrm{OK}+\mathrm{H}_{2} \mathrm{~S} \text {. }
$$




$$
\mathrm{CO}\left(\mathrm{OC}_{6} \mathrm{H}_{5}\right)_{2}+2 \mathrm{NaOC}_{2} \mathrm{H}_{5}=\mathrm{CO}\left(\mathrm{OC}_{2} \mathrm{H}_{5}\right)_{2}+2 \mathrm{NaOC}_{6} \mathrm{H}_{5} \text {. }
$$

Reibt man Diphenylcarbonat und Natriumäthylat in einer kalten Reibschale zusammen, so findet nach kurzer Zeit eine heftige Reaktion unter theilweiser Schwärzung und so starker Wärmeentwicklung statt, dass das gebildete Diäthylcarbonat vollständig verdampft. Deshalb musste das folgende Verfahren innegehalten werden, welches sich jedenfalls auch für die am Anfange beschriebene Ueberführung des Diphenylcarbonats in Dithioäthylcarbonat eignen wird.

Ein Molekül Diphenylcarbonat wurde in einem Kolben mit absolutem Aether übergossen und nach und nach mit überschüssigern, absolut trocknem Natriumäthylat (etwa drei Molekülen) behandelt. Oefteres Kühlen hielt die Reactionstemperatur niedrig. Anfangs trat Lösung ein, bald darauf Abscheidung von Phenolnatrium. Wasser löste aus dem Produkte Phenolnatrium und schied eine ätherische Schicht $a b$, welche mit Wasser gewaschen und über Kalk getrocknet wurde. Durch Rectification erhielt ich in ziemlich guter Ausbeute Diäthylcarbonat vom Siedepunkt $126^{\circ}$ bis $128^{\circ}$. Bei Mangel an Natriumätbylat bilden sich nicht unbeträchtliche Mengen eines höher siedenden Produktes, welches wahrscheinlich Aethylphenylcarbonat $\mathrm{CO}_{\mathrm{OC}_{2} \mathrm{H}_{5}}^{\mathrm{OC}_{5} \mathrm{H}_{5}}$ enthält.

Ich habe vorhin an einigen Schlüssen erläutert, dass durch eine Reihe wie die oben aufgeführte die Wechselbeziehungen der Körper zu einander auf einfache Weise klar in die Augen springen. Es würde deshalb wohl keine verlorene Mühe sein, die hauptsächlichsten Körper der organischen und vielleicht auch der Mineralchemie, welche durch Metalle ersetzbaren Wasserstoff enthalten, an diese Reihe anzuschliessen.

Nur zur Erläuterung dieses Gedankens soll folgende, ganz fragmentarische Reihe dienen: Alkoholbasen, Aniline, Acetamid, Acetanilid, Alkohole, Merkaptane, Phenole, Thiophenole, Carbonsäuren, Sulfosäuren. 
Also auch in dieser Reihe nehmen die basischen Eigenschaften von links nach rechts $a b$, die sauren $z u$.

\section{An hang.}

Bildung von Orthobenzophenonoxyd aus Phenyl-

$$
\text { salicylat. }
$$

Bemerkenswerth ist das Verhalten des Phenylsalicylats in der Hitze. Während es beim Erwärmen im zugeschmolzenen Rohr auf $240^{\circ}$ unverändert blieb, zersetzte es sich beim Destilliren unter gewöhnlichem Druck vollständig. Durch Rektificiren des Destillats wurde neben einer grossen Menge Phenol ein syrupöser Rückstand gewonnen, welcher bei längerem Erhitzen am Rückflusskühler O-Benzophenonoxyd lieferte.

Von der Untersuchung des syrupösen Zwischenproduktes absehend und nur die Darstellung von Benzophenonoxyd im Auge behaltend, modificirte ich den Versuch in folgender Weise:

In einem mit aufsteigendem Kühlrohr und sich daranschliessendem Gasableitungsrohr versehenen Kölbchen wurde Phenylsalicylat im Sieden erhalten. Anfangs spaltete sich nur wenig Kohlensäure, aber viel Phenol ab, welches ich von Zeit zu Zeit bis $190^{\circ}$ abdestillirte. Beim darauffolgenden Wiedererhitzen fand immer eine verhältnissmässig starke Kohlensäureentwicklung statt. Liess die Phenol- und Kohlensäureentwicklung nach, was bei 30 bis 40 Grm. Ester nach 9 bis 10 Stunden der Fall war, so wurde der dunkle, krystallinisch erstarrende Rückstand rektificirt. Das Thermometer stieg schnell auf $360^{\circ}$, und unter Zurücklassung von wenig Kohle destillirte ein gelblicher, sofort erstarrender Körper vom Schmelzpunkt $160^{\circ}$. Dieser krystallisirte aus Alkohol in langen, seideglänzenden Nadeln vom Schmelzpunkt $170^{\circ}$ bis $171^{\circ}$, war mit Wasserdämpfen flüchtig und löste sich beim Schütteln mit geschmolzenem Kaliumhydroxyd vollständig auf. Die in Wasser gelöste Kalischmelze wurde angesäuert, mit Aether extrahirt, der Aether mit Ammonium- 
carbonatlösung geschüttelt und hierauf verdunstet. Zurück blieb Phenol, welches mit Brom Tribromphenol lieferte. Die Ammoniumcarbonatlösung gab nach dem Ansäuern an Aether Salicylsäure ab.

Der erhaltene Körper ist nach dem Vorstehenden unzweifelhaft O-Benzophenonoxyd $\mathrm{C}_{6} \mathrm{H}_{4} \backslash \mathrm{CO}>\mathrm{C}_{6} \mathrm{H}_{4}$, welches aus dem Phenylsalicylat nach folgender Gleichung entstehen muss:

$$
2 \mathrm{C}_{6} \mathrm{H}_{4}-\mathrm{OH}=\mathrm{OH}_{6} \mathrm{H}_{5}=\mathrm{C}_{13} \mathrm{H}_{8} \mathrm{O}_{2}+\mathrm{CO}_{2}+2 \mathrm{C}_{6} \mathrm{H}_{5} \mathrm{OH} .
$$

Ich erhielt bei einem Versuche neben 13 Grm. Phenol 13,9 Grm. rohes Benzophenonoxyd. Die obige Gleichung verlangt 13,6 Grm. Benzophenonoxyd auf 13 Grm. Phenol.

Da Phenylsalicylat leicht und in fast theoretischer Ausbeute $\mathrm{zu}$ gewinnen ist, betrachte ich dasselbe als das beste Ausgangsmaterial zur Herstellung von Orthobenzophenonoxyd.

Wie schon erwähnt, geht der Entstehung dieses Körpers die eines $Z$ wischenproduktes voran. Nimmt man als solches Salicylid an, so gestaltet sich die Erklärung des Vorganges sehr einfach:

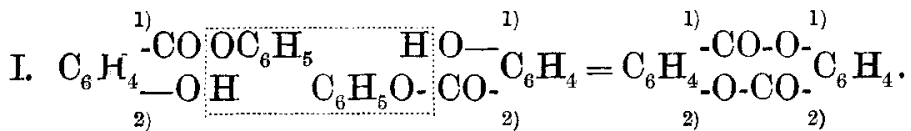

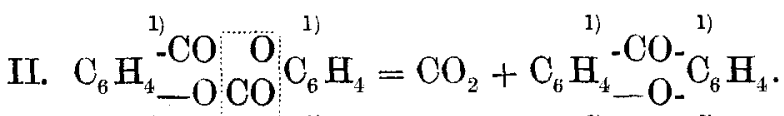

$$
\begin{aligned}
& \text { 2) } \\
& \text { 2) } \\
& \text { 2) 2) }
\end{aligned}
$$

Durch die letzte Gleichung erklärt auch W. H. Perkin ${ }^{1}$ ) die Bildung von Benzophenonoxyd aus Salicylsäure und Acetanhydrid.

Da mir kein Salicylid zu Gebote stand, so konnte ich leider nicht prüfen, $a b$ dieses beim Erhitzen für sich oder mit Phenol wirklich Benzophenonoxyd liefert. Das Gelingen

1) Ber. Berl, chem. Ges. 16, 339 . 
480 Seifert: Einwirkung von Natriummerkaptid etc.

dieser Umwandlung würde ein Beweis für die in obigen Gleichungen angenommene doppelte Salicylidformel sein. Aber unwahrscheinlich bliebe dann immer noch die merkwürdige Art der Abspaltung von Kohlensäure, wie sie in vorstehender Gleichung II angedeutet ist. Ich glaube vielmehr, dass diese Abspaltung im Sinne folgender Gleichung verläuft:

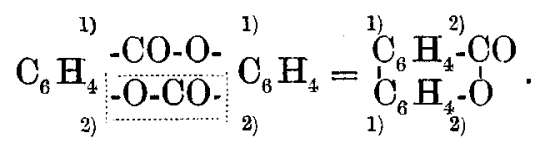

Diese letzte Formel giebt auch E. Spiegler ${ }^{1}$ ) dem Benzophenonoxyd wegen der Unfähigkeit desselben, mit Hydroxylamin und Phenylhydrazin die für Ketone charakteristischen Verbindungen zu bilden. Durch Oxydation des Benzophenonoxyds wird sich die Frage nach seiner Constitution entscheiden lassen. Gehört dasselbe zu den Lactonen, so wird durch Oxydation Phtalsäure entstehen.

Die Reaction, durch welche Phenylsalicylat in Benzophenonoxyd verwandelt wird, scheint auch für Phonylester anderer Oxysäuren zu gelten. Citronensäurephenylester spaltet beim Erhitzen mit grosser Leichtigkeit Phenol und Kohlensäure ab, und auch Oxybenzoesäurephenylester erleidet eine ähnliche Umwandlung, mit deren Studium Herr stud. Retter beschäftigt ist.

Ich beabsichtige $\mathrm{zu}$ entscheiden, ob die Ueberführung einer Oxy- oder Amidosäure in ihren Phenylester und das Erhitzen desselben eine Methode ist zur Darstellung von Condensationsprodukten der Zusammensetzung $\mathrm{R}_{-\mathrm{O}}^{\mathrm{II}-\mathrm{CO}}>$ und $\mathrm{R}_{--\mathrm{NH}}^{\mathrm{II}-\mathrm{CO}}>$.

1) Ber. Berl. chem. Ges. 17, 807. 